\title{
Final Report: Deconvolution of Adaptive Optics Images of Titan, Neptune, and Uranus
}

S. Gibbard, F. Marchis

December 20, 2002

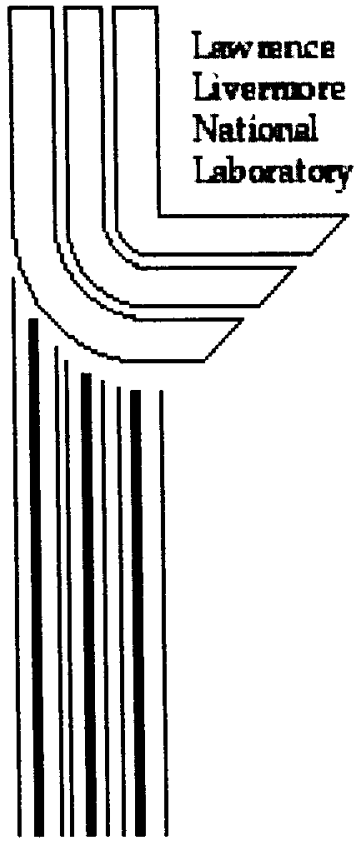




\section{DISCLAIMER}

This document was prepared as an account of work sponsored by an agency of the United States Government. Neither the United States Government nor the University of California nor any of their employees, makes any warranty, express or implied, or assumes any legal liability or responsibility for the accuracy, completeness, or usefulness of any information, apparatus, product, or process disclosed, or represents that its use would not infringe privately owned rights. Reference herein to any specific commercial product, process, or service by trade name, trademark, manufacturer, or otherwise, does not necessarily constitute or imply its endorsement, recommendation, or favoring by the United States Government or the University of California. The views and opinions of authors expressed herein do not necessarily state or reflect those of the United States Government or the University of California, and shall not be used for advertising or product endorsement purposes.

This work was performed under the auspices of the U. S. Department of Energy by the University of California, Lawrence Livermore National Laboratory under Contract No. W-7405-Eng-48.

This report has been reproduced directly from the best available copy.

Available electronically at http://www.doe.gov/bridge

Available for a processing fee to U.S. Department of Energy and its contractors in paper from

U.S. Department of Energy

Office of Scientific and Technical Information

P.O. Box 62

Oak Ridge, TN 37831-0062

Telephone: (865) 576-8401

Facsimile: (865) 576-5728

E-mail: reports@adonis.osti.gov

Available for the sale to the public from

U.S. Department of Commerce

National Technical Information Service 5285 Port Royal Road

Springfield, VA 22161

Telephone: (800) 553-6847

Facsimile: (703) 605-6900

E-mail: orders@ntis.fedworld.gov

Online ordering: http://www.ntis.gov/ordering.htm

OR

Lawrence Livermore National Laboratory

Technical Information Department's Digital Library

http://www.llnl.gov/tid/Library.html 
Final Report:

\section{Deconvolution of Adaptive Optics Images of Titan, Neptune, and Uranus}

Work done during the period of July 18 2000-September 302002 by Franck Marchis, UC Berkeley under IUT agreement No. B522320

Report prepared for Seran Gibbard, Institute for Geophysics and Planetary Physics at Lawrence Livermore National Laboratory

Date of report: December 20, 2002 


\section{Deconvolution of adaptive optics images of Titan, Neptune, and Uranus}

This project involved images of Titan, Neptune, and Uranus obtained using the 10-meter W.M. Keck II Telescope and its adaptive optics system. An adaptive optics system corrects for turbulence in the Earth's atmosphere by sampling the wavefront and applying a correction based on the distortion measured for a known source within the same isoplanatic patch as the science target (for example, a point source such as a star). Adaptive optics can achieve a 10-fold increase in resolution over that obtained by images without adaptive optics (for example, Saturn's largest moon Titan is unresolved without adaptive optics but at least 10 resolution elements can be obtained across the disk in Keck adaptive optics images).

The adaptive optics correction for atmospheric turbulence is not perfect; a point source is converted to a diffraction-limited core surrounded by a 'halo'. This halo is roughly the size and shape of the uncorrected point spread function one would observe without adaptive optics. In order to enhance the sharpness of the Keck images it is necessary to apply a deconvolution algorithm to the data. Many such deconvolution algorithms exist, such as maximum likelihood and maximum entropy. These algorithms suffer to various degrees from noise amplification and creation of artifacts near sharp edges ('ringing'). In order to deconvolve the Keck images I have applied an algorithm specifically developed for observations of planetary bodies, the myopic deconvolution algorithm MISTRAL ('Myopic Iterative STep-preserving Restoration ALgorithm') (Conan et al. 1998, 2000). MISTRAL was developed by ONERA (Office National d'Etudes et de Recherches Aerospatiales) and has been extensively tested on simulated and real AO observations, including observations of Titan (Coustenis et al.2001), Io (Marchis et al.2002, 2001), and asteroids (Hestroffer 
et al.2001, Rosenberg et al.2001, Makhoul et al.2001). Compared to more classical methods, MISTRAL avoids noise amplification and ringing artifacts, and better restores the initial photometry (Conan et al.1998). MISTRAL uses a stochastic approach to finding the best image reconstruction, using information about the object and the PSF.

The results from my work on deconvolution of Titan and Uranus images are shown on these websites: http://astron.berkeley.edu/ fmarchis/Science/Uranus/NIRC2/ and http://astron.berkeley.edu/ fmarchis/Science/TitanAO/Simu/ (NOTE: these websites are for internal use only, not for general distribution). Copies of these websites are attached to this report. The general conclusions from this deconvolution effort is that MISTRAL does an excellent job of exhancing the sharpness of the data and preserving the photometry. The continued use of this algorithm for deconvolution of adaptive optics data is strongly suggested.

\section{References:}

Conan, J.-M., T. Fusco, L.M. Mugnier, F. Marchis, C. A. Roddier and F.J. Roddier. 2000. Deconvolution of adaptive optics images: from theory to practice. Proc. SPIE Vol. 4007, p. 913-924, Adaptive Optical Systems Technology, Peter L. Wizinowich; Ed.

Conan, J.-M., L.M. Mugnier, T. Fusco, V. Michau, and G. Rousset. 1998. Myopic deconvolution of adaptive optics images by use of object and point-spread function power spectra. Applied Optics 37, 4616-4622.

Coustenis, A., E. Gendron, O. Lai, J.-P. Veran, J. Woillez, M. Combes, L. Vapillon, T. Fusco, L. Mugnier and P. Rannou. 2001. Images of Titan at 1.3 and $1.6 \mu \mathrm{m}$ with Adaptive Optics at the CFHT. Icarus 154, 501-515.

Hestroffer, D., F. Marchis, T. Fusco, and J. Berthier. 2001. Adaptive optics observations of asteriod 216 Kleopatra. Submitted as a Research Note to A\%A.

This work was performed under the auspices of the U.S. Department of Energy by the University of California, Lawrence Livermore National Laboratory under Contract No. W-7405-Eng-48. 
Makhoul, K. A. Storrs, J.-M. Conan, B. Zellner, E. Wells, F. Vilas, R. Landis, and M. Gaffey. 2001. Results from the HST Main Belt Asteroid Survey. American Astronomical Society Meeting 199, \#63.02.

Marchis, F., I. de Pater, A. Davies, H.G. Roe, P. Descamps, D. Le Mignant, and B.A. Macintosh. 2002. High-resolution Keck adaptive optics imaging of violent volcanic activity on Io. submitted to Icarus.

Marchis, F., R. Prangé, and T. Fusco, 2001. A survey of Io's volcanism by adaptive optics observations in the $3.8-\mu \mathrm{m}$ thermal band (1996-1999). J. Geophys. Res. 106, 141-160.

Rosenburg, A., A. Stoors, J.-M. Conan, D.W. McCarthy, R.P. Binzel, J. Drummond, M. Gaffey, K. Hege, L. Lebofsky, P. Thomas, E. Wells, and B. Zellner. 2001. Mineralogical Mapping of Asteroid 4 Vesta. American Astronomical Society Meeting 199, \#63.01. 


\section{Simulation of Titan images with MISTRAL}

This web page summarizes a simulation of deconvolution of Titan images based on real observations of the satellite taken at the 10-meter W.M. Keck Telescope in Kp band (centered at 2.1 microns) with the NIRSPEC camera in February 2001.

\section{Creation of the image}

On the 22nd of February 2001, the angular diameter of Titan was 0.8 , i.e. 48 pixels across with the NIRSPEC 16.8 mas (milliarcsecond) pixel size. The data were taken in Kp band (1.995-2.292 microns) with a total integration time of 60 seconds. Statistical study on the image indicates a total count (data numbers) of 1.45E7 and a background noise comparable with a Poisson/Gaussian statistics with a variance of 40 data numbers. During the observing nights 4 PSF (point spread function) stars were observed. Their full width at half maximum (FWHM) varies from 52 to 57 mas.

The artificial Titan image (also called the 'perfect image') was generated using a radiative transfer model that simulates the absorption, reflection and scattering of photons from Titan's atmosphere and a constant-albedo surface (Toon et al 1989). We have previously used this model to simulate Titan's atmosphere (Gibbard et al 1999); the model has also been applied to Titan by Toon et al (1992), and Hutzell et al $(1993,1996)$. The 'perfect image' produced by the model was convolved with one of the four observed PSF's. Poisson noise and Gaussian noise were added mimicking as well as possible the observed image obtained on February 2001.

Seran Gibbard created the perfect image and chose the PSF. She sent Franck Marchis the final convolved and noisy image without given him any clues about the structure of the perfect Titan image or which PSF was used.

\section{Deconvolution}




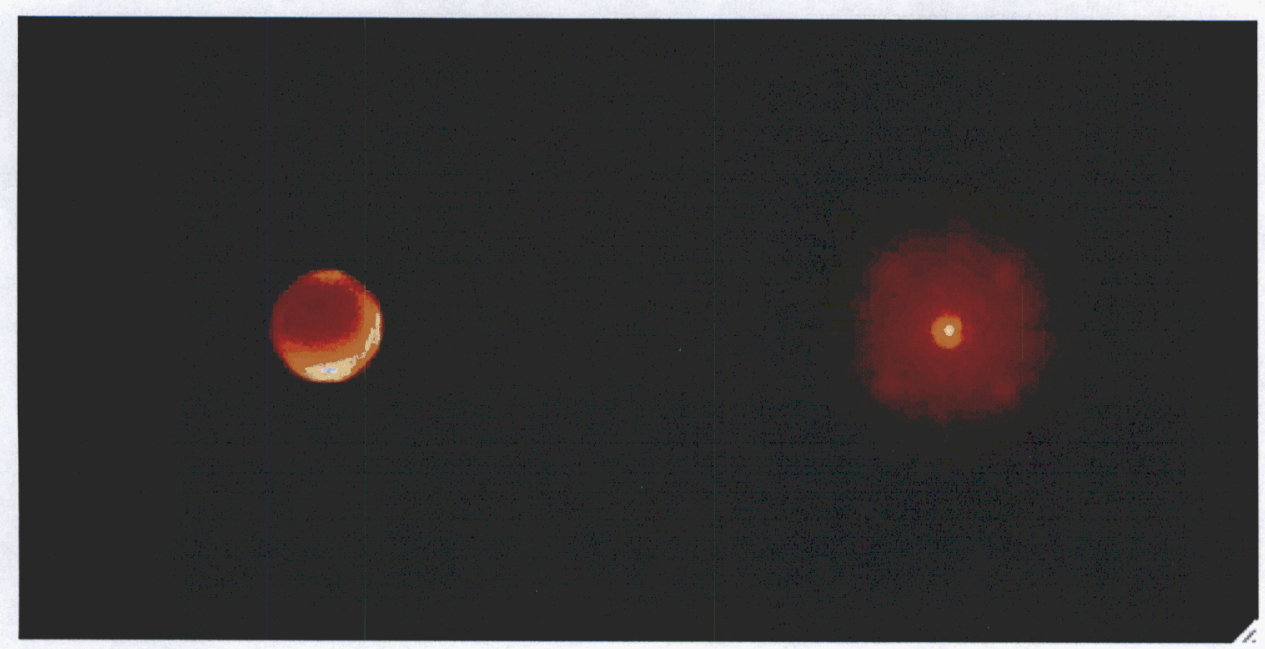

Fig. 1: Convolved and Noisy image of Titan (left), logarithmic display of the mean PSF (right) used for the deconvolution.

\subsection{PSFs and PSD}

The four PSF's which were taken during the observing night were used to calculated a mean PSF and the Power Density Spectrum (PSD). The PSD indicates the variation of the PSF in Fourier Space. A display of the Optical Tranfer Function (OTFs) of each PSF can be found here (the solid line is the OTF of the mean PSF, and the dashed lines are the OTF of each PSF). Logarithmic display of each PSF and its FWHM in Fig. 2 clearly indicates the variation of the AO correction.

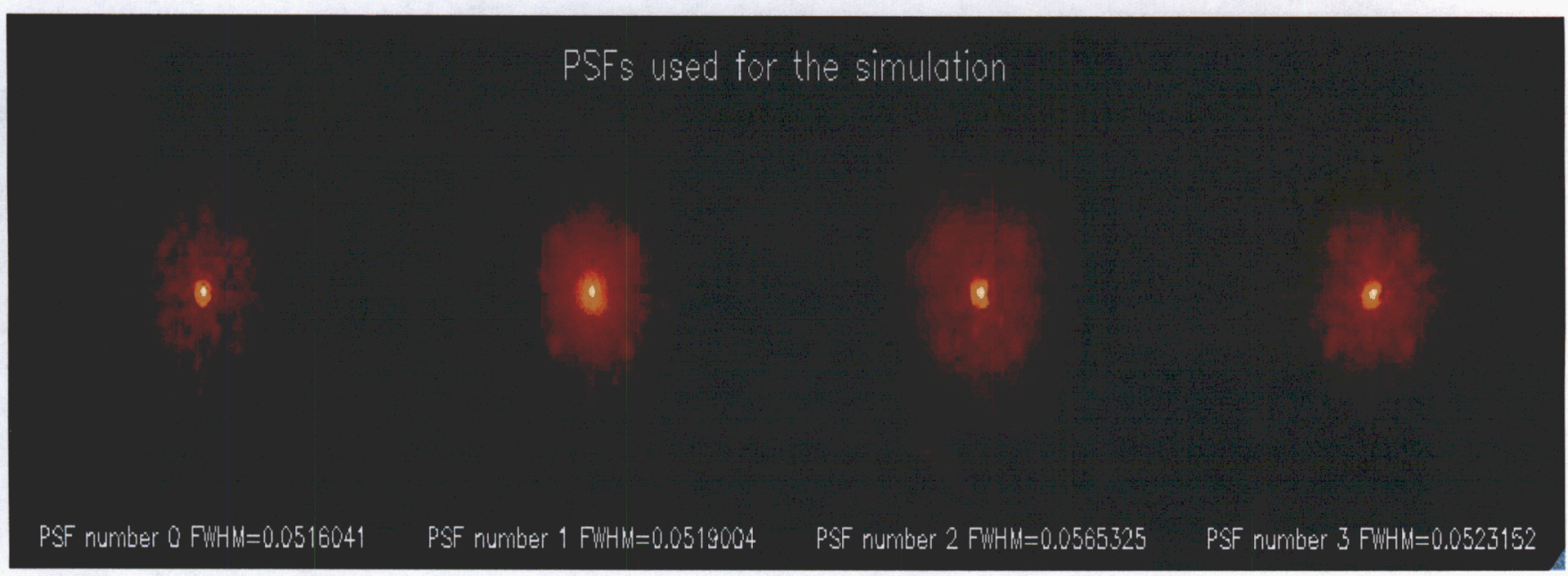

Fig. 2: Four PSF's used for the deconvolution. One can notice the variability of the AO correction which clearly modifies the shape and the FWHM of PSF's, the sharp cut due to the small size of the detector and the spectrograph slit.

\subsection{Myopic Deconvolution using MISTRAL}

Deconvolution using MISTRAL is not simple. Details about the algorithm and the way to use it can be found in several proceedings (Conan et al., 1998; Conan et al., 2000; Fusco et al., 2002) and Ph.D.thesis 
(Fusco 2000; Marchis 2000). The optimal regularization variables must be determined and these parameters (called h, s, and hpsf) depend on the statistics of the image, the shape of the body, and the quality of correction of the AO system. There is no direct rule to get the best set of parameters for each dataset. The deconvolution is performed with a high number of iterations which will make obvious any under-regularized or over-regularized deconvolved frames. The complete set of deconvolved frames can be found here. Figure 3 displays two extremely under- and over- regularized frames and one frame which seems to be good. Deconvolution using MISTRAL is not simple. The optimal regularization variables must be determined and these parameters (called h, s, and hpsf) depend on the statistics of the image, the shape of the body, and the quality of correction of the AO system. There is no direct rule to get the best set of parameters for each dataset. The deconvolution is performed with a high number of iterations which will make obvious any under-regularized or over-regularized deconvolved frames. The complete set of deconvolved frames can be found here. Figure 3 displays two extremely under- and over- regularized frames and one frame which seems to be good.

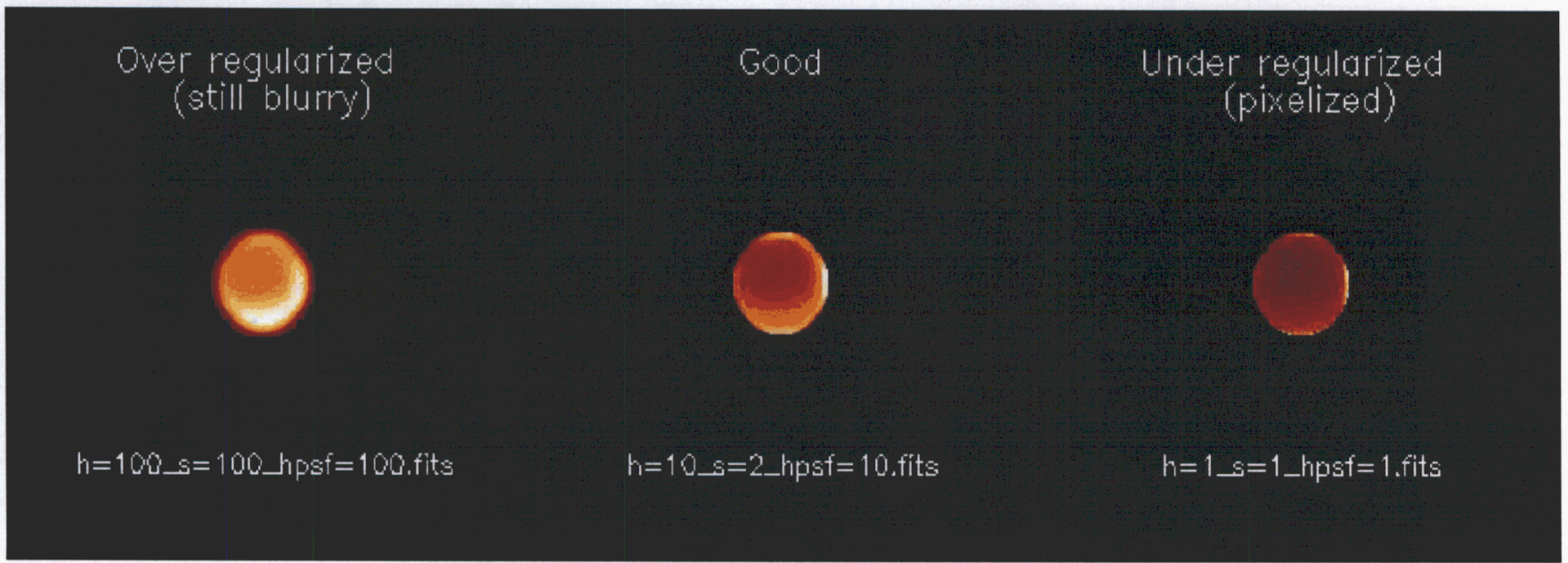

Fig. 3: Under regularized (maximum likelihood prior is dominant) frame (right), over regularized frame (the regularized factor is over estimated) on the left. The frame displayed at the center represents one of the possible good deconvolved frame.

As shown in the set of figures, there are several apparently good frames that can be chosen. Looking at the residual background of some of the frames one can see that it is minimized for a very few of them. The frame corresponding to $\mathbf{h}=\mathbf{1 0}, \mathbf{s}=\mathbf{5} \& \mathbf{h p s} \mathbf{f}=\mathbf{1 0 0}$ seems to be the best one. The frame $h=10, s=5 \&$ $\mathrm{hpsf}=10$ is a second possible good frame.

\section{Comparison with Perfect Image}

\subsection{Images}

After the deconvolution was finished and the best frames chosen, S. Gibbard sent to F. Marchis the original 'perfect' Titan image. The comparison between the perfect image and the chosen 'best' deconvolved image is shown in Fig. 4. 


\section{Simulation of deconvolution with MSTRAL Titan abserved with NIFSPEC AO}
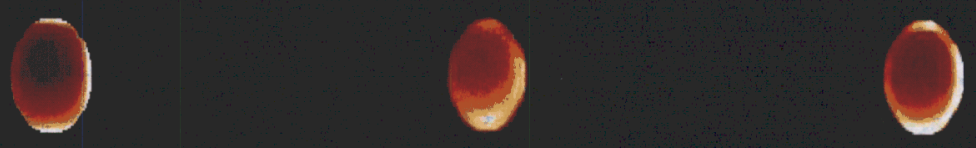

Fig. 4: Perfect image and comparison with deconvolved images.

The asymmetry of the haze brightness (slightly brighter in the South-West pole) is recovered. The intensity on the disk and on the limb seems similar.

Vertical and Horizontal cut profiles indicate that the general profile of the intensity is maintained with a preference for the $\mathbf{h}=\mathbf{1 0}, \mathbf{s}=\mathbf{5} \& \mathbf{h p s f}=\mathbf{1 0 0}$ image (see Fig. 5)

Best Frame $(h=10, s=5 \&$ hpsf=100)

2nd Best Frame $(h=10, s=5 \&$

$$
\text { hpst }=10 \text { ) }
$$




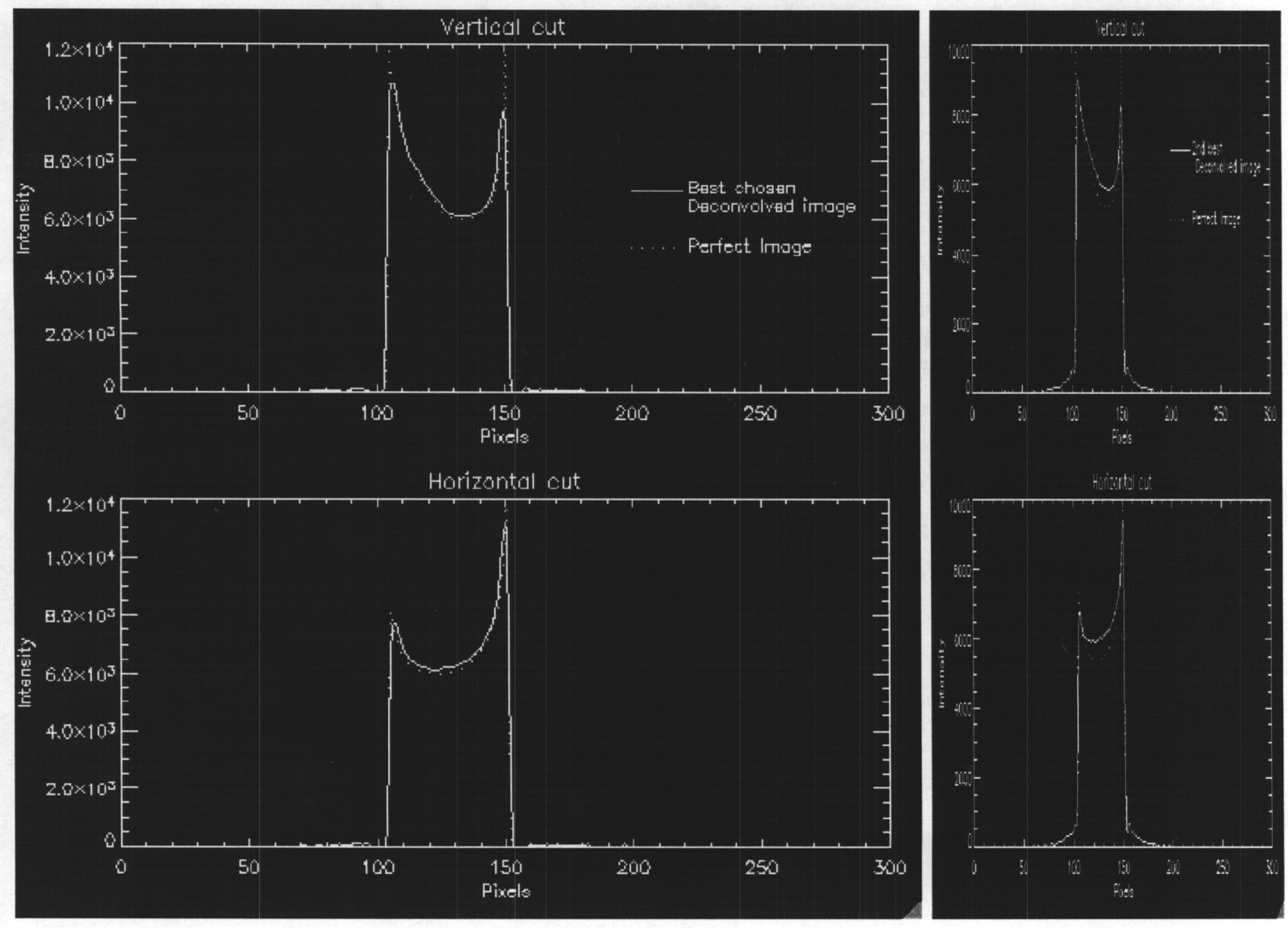



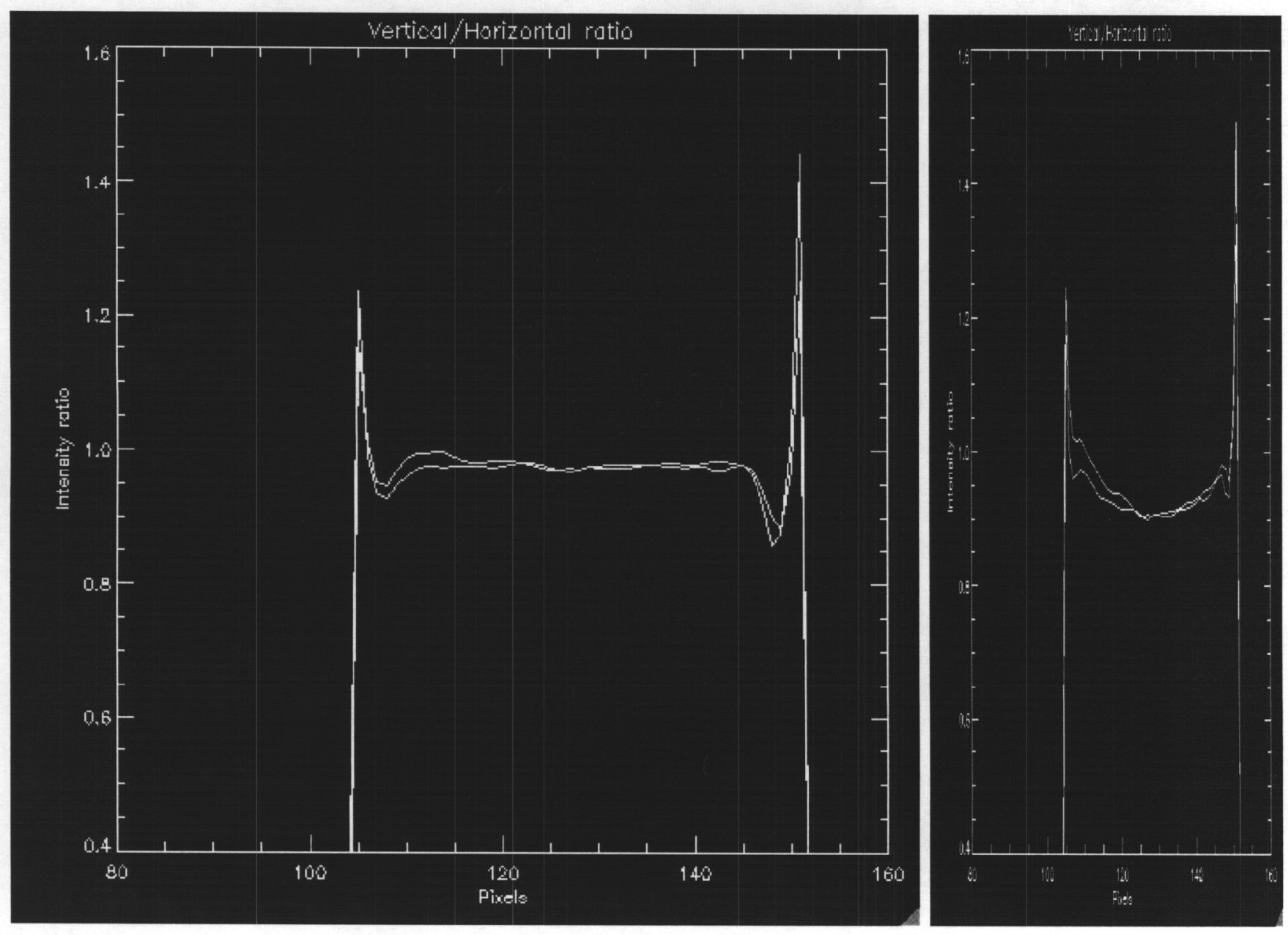

Table 1: Intensity comparison of the deconvolved and perfect Titan images.

The comparative intensity ratio between the best and second best frame clearly indicates that the first estimate is better with a mean ratio on the disk of 0.98 . On the second image the ratio varies from 1.05 to 0.92 on the disk. The edge is not as well reconstructed as the disk. The ratio reaches 1.45 for the best image. It is interesting to notice that any feature located at more than 5 pixels from the limb and with a contrast greater than $5 \%$ will be easily detected with MISTRAL. Such features (methane clouds) have been detected in Titan high-resolution images by several observers (Roe et al 2002, Brown et al 2002, Gibbard et al 2002).

\subsection{PSFs}

Since MISTRAL is a myopic deconvolution method it also recovers the best PSF for the deconvolved frame. Figure 5 displays both the reconstructed PSF and the median PSF. The PSF which was chosen by Seran for the convolution was number 2 which has a FWHM=56.5 mas. The FWHM of the mean PSF was better with 52.8 mas. MISTRAL seems to recover quite well the PSF used for the deconvolution since it has a FWHM of 55.8 mas (for the best frame). One can also notice that the algorithm tends to smooth the PSF, removing the sharp cut introduced by the small detector size. 


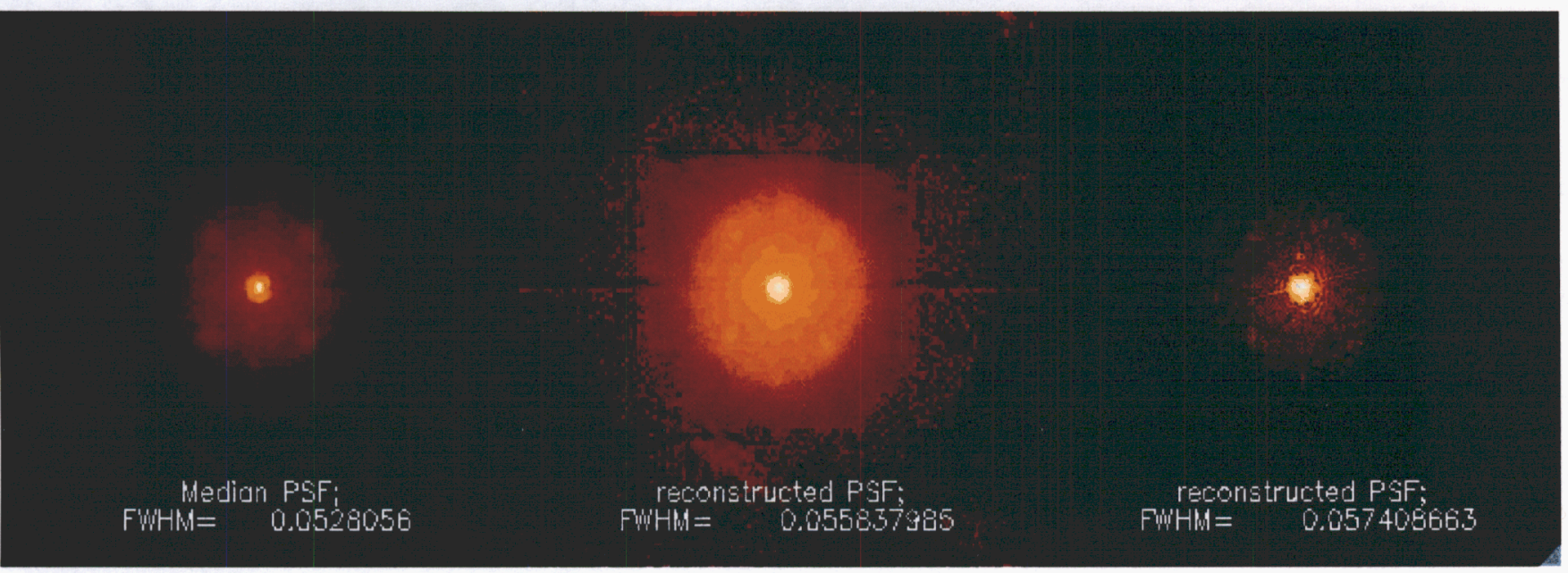

Fig 5: Median PSF and two PSF reconstructed by MISTRAL.

\section{Conclusion}

These results clearly demonstrate the capability of MISTRAL to deconvolve Titan images and recover the photometry with a good accuracy. The main difficulty for this kind of data is the presence of a bright limb introduced by Titan's atmosphere and that should not be considered as a Gibbs artifact. Even if the limb profile is not very well reconstructed, the disk is in comparison recovered with an accuracy better than $8 \%$.

This simulation was performed honestly. Seran Gibbard and Franck Marchis are not directly involved in the development of MISTRAL. Our goal was to show here the potential of MISTRAL and to check through this simulation the accuracy of the Feb. 2001 data measurement.

\section{To be done (in no special order)}

- Comparison with other deconvolution methods (Lucy-Richardson, wavelet...)

- Check deconvolution and Estimate of the Albedo error on the Gibbar et al. (2002, submitted to Icarus) paper data

- Simulation of Io with hot spots in L-M band

- Check deconvolution with bright polar feature (limit of detection of clouds)

- deconvolve images with polar clouds

- write a paper with all the simulations.... (Io, Titan, Uranus, stellar field...)

- .... 


\section{Additional links}

- Uranus observed with NIRC2 (deconvolution with MISTRAL)

- Io observed with NIRC2 (deconvolution with MISTRAL)

\section{References}

- Brown, M.E., A.H. Bouchez, and C.A. Griffith. 2002. Clouds at the South Pole of Titan. American Astronomical Society, DPS meeting \#34, \#22.10

- Conan, J.-M., Fusco, T., Mugnier, L.M., Kersale, E., Michau, V.. 1998. Deconvolution of Adaptive Optics images with imprecise knowledge of the PSF: Results on astronomical objects. ESO/OSA Topical Meeting, Astronomy with Adaptive Optics. Conan J.-M., T. Fusco, L.M. Mugnier, F. Marchis, C. Roddier, F. Roddier. 2000. Deconvolution of adaptive optics images: from theory to practice, SPIE proceeding, Astronomy 2000, Munich, March 2000, 4007, 913-924.

- Fusco, T., 2000. Correction partielle et anisoplanétisme en Optique Adaptative: Traitement a posteriori et Optique Adaptative Multiconjugué, Université de Nice, October 62000.

- Fusco T., L. Mugnier, J.-M. Conan, F. Marchis, G. Chauvin, G. Rousset, A.-M. Lagrange, D. Mouillet and F. Roddier. 2002. Deconvolution of astronomical images obtained from ground-based telescopes with Adaptive Optics, in Proc. SPIE, Vol. 4839, in press.

- Gibbard, S.G., B.A. Macintosh, C.E. Max, I. de Pater, F. Marchis, H.G. Roe, D.S. Acton, O. Lai, P.L. Wizinowich, P. Stomski, E.F. Young, and C.P. McKay. 2002. Observations of Titan at 2 microns with the W.M. Keck Telescope adaptive optics system. Submitted to Icarus.

- Gibbard, S.G., B. Macintosh, D. Gavel, C.E. Max, I. de Pater, A.M. Ghez, E.F Young, and C.P. McKay. 1999. Titan: high-resolution speckle images from the Keck Telescope. Icarus 139, 189-201.

- Gibbard, S.G., B. Macintosh, D. Gavel, C.E. Max, I. de Pater, H.G. Roe, A.M. Ghez, E.F Young, and C.P. McKay. 2002. Titan: 2 micron surface albedo, stratospheric haze optical depth and tropospheric clouds in 1996-1998. Submitted to Icarus.

- Hutzell, W.T., C.P. McKay, and O.B. Toon. 1993. Effects of time-varying haze production on Titan's geometric albedo. Icarus 105, 162-174.

- Hutzell, W.T., C.P. McKay, O.B. Toon, and F. Hourdin. 1996. Simulations of Titan's brightness by a two-dimensional haze model. Icarus 119, 112-129.

- Marchis, F., Imagerie à haute résolution angulaire des objets du système solaire en Optique Adaptative. Application à l'étude du volcanisme de Io, Thèse de l'Université P. Sabatier, Toulouse III, October 42000.

- Roe, H.G., I. de Pater, B.A. Macintosh and C.P. McKay. 2002. South polar clouds on Titan imaged with adaptive optics on the Gemini and Keck telescopes. Ap. J., in press.

- Toon, O.B., C.P. McKay, T.P. Ackerman, and K. Santhanam. 1989. Rapid calculation of radiative heating rates and photodissociation rates in inhomogenous multiple scattering atmospheres. J.G.R. 94, 16287-16301.

- Toon, O.B., C.P. McKay, C.A. Griffith, and R.P. Turco. 1992. A physical model of Titan's aerosols. Icarus $95,24-53$. 


\section{F. Marchis}

- $\quad$ Last Modified: Mon Nov 25 20:28:PDT 2002 


\section{Uranus observed with NIRC2 (deconvolution with MISTRAL)}

The goal of this webpage is to summarize briefly the results of deconvolution with MISTRAL applied on the first NIRC-2 image. Scientific analysis will be done "soon".

\section{Description of the data}

Uranus was observed in Ks band on 18th december 2001 at 04:50 UT with the NIRC-2 camera in Ks band (central wavelength 2.147 microns). The field of view of each frame is $10.24 \mathrm{arcsec}$, with a theoretical pixel size of 0.01 mas. Individual integration time on each frame was $60 \mathrm{~s}$. The deconvolved frame is the result of the average of 5 frames. Airmass during observations was 1.62.

We had several troubles to find a suitable PSF for the deconvolution. The SAO star taken in the same wavelength range and nearby the target (n0155.fits) is clearly elongated, so useless for the deconvolution. We finally used n0275. fits (PSF for Io observation) observed 1 hour after the Uranus observation, in $\mathrm{Kp}$ band and with an airmass of 1.16. quite challenging for the deconvolution, even if MISTRAL is a myopic method....

\section{Basic-processed image}

To perform a faster deconvolution, I did not deconvolve the entire $1700 * 1700$ pixels frame, I cropped a $800 * 800$ pixel frame centered on the Uranus disk. The left part of the figure (see below) displays this image. Two internal satellites of Uranus are detectable with a peak S/N of 4 for Cressida and less than 2 for Desdemona. On the off-centered image (on the left), several other moons are visible in the north part of the image. Puck is clearly visible with a peak $S / N$ of 8 , Portia also with a $S / N=6$. Rosalind and Belinda are marginally detected with a S/N of 2-4. Note also tha each inner satellite appears with a trail due to the smearing effect introduced by their motion during the observation. Identification was done using the Ring-Node tool (JPL ephemeris see .ps file).

Three first rings are easily visible on the south Anse. A fourth one is barely visible. The disk of the planet is dark due to the methane absorption. The limb is anyway brighter because of a limb-brightening effect introduced by the high altitude haze.

The observing artifact seen in the NIRSPEC images and introduced suposely by the AO (see de Pater et al., 2002, in press, Icarus) seems to be also present on this data (the Northern rings are double), but with a lower intensity. Comparison with the previous observation (intensity, shape) should be done. The PSF is displaid in the inset of the figure (log scale). SR cannot be calculated, but the FWHM is estimated to 4.9 pixels (49 mas). 


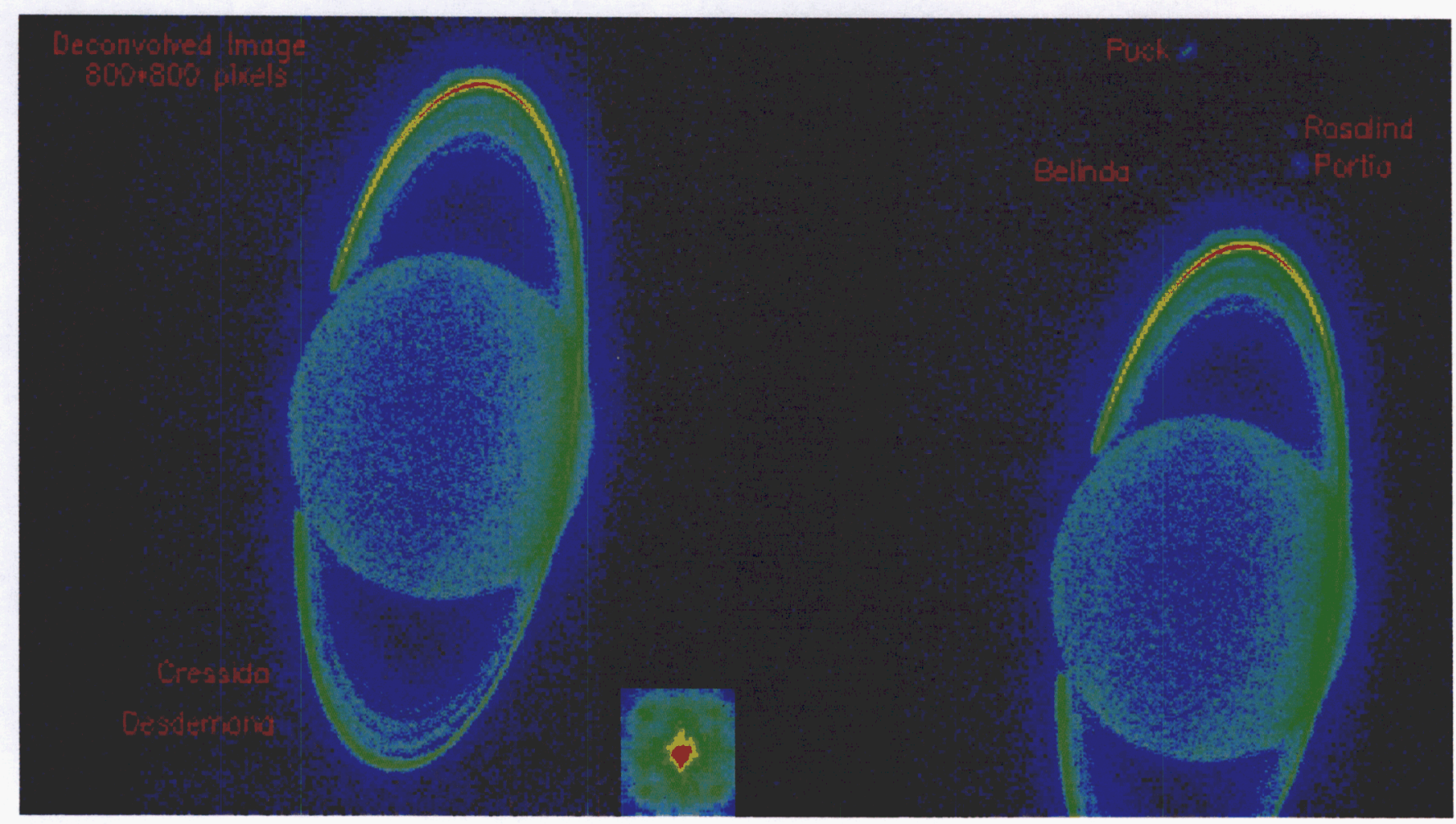

\section{First deconvolved images}

I used MISTRAL to deconvolve the $800 * 800$ frames. Deconvolution was performed in classical mode and with myopic option. Since I do not have a several PSF frames, the variation of the PSF was estimated assuming a pure Poisson noise statistic. The following images is the result of deconvolution using several different parameters. They are display with $h$ decreasing. $h$ is the main regularisation parameter of MISTRAL. It balances the pure maxlikehood solution (assuming a white non stationary Gaussian noise) with an object prior which avoid the usual ringing artifact. The second parameter $(s)$ is the threshold limit of the L1-L2 norme. Finally the last parameter, calledhpsf, defined the weight of the myopic criterion which is related to the unstability of the PSF (see Conan et al., SPIE, 2000, Fusco, $2000, \mathrm{PhD}$. or Marchis, 2000,PhD.) for details). When $h$ is greater than 40 , the image is clearly over-regularized (it is still blurry), when $h$ is lower than 3 , the image is sub-regularized and the maxlikehood artifact are easily visible (image is pixelized). The best compromised image is between these values. $h=4$ is clearly the best image obtained.

The myopic option is clearly efficient on this type of image even if the variability of the PSF is an rought estimate. The Epsilon ring on the myopic image is complete. The reconstructed PSF is slightly elongated in the $\mathrm{N}-\mathrm{S}$ direction (FWHM=6.1 pixels) and the AO artifact feature is minimized on the deconvolved data.

The four rings are detected with an higher S/N (more than 1E8 for Epsilon). Feature variation as seen in the ADONIS image are visible (see Marchis, 2000, $\mathrm{PhD}$ thesis or this link). Cressida is also visible with a local S/N higher than 1E7. The deconvolution method kept the elongated shape of the asteroid, avoiding the classical pixelisation effect introduced by a classical Lucy-Richardson. Cressida was never detected on our previous ADONIS) observation, and Puck S/N achieved by this observation was less than 200 for the detection of Puck in $\mathrm{H}$ band. 

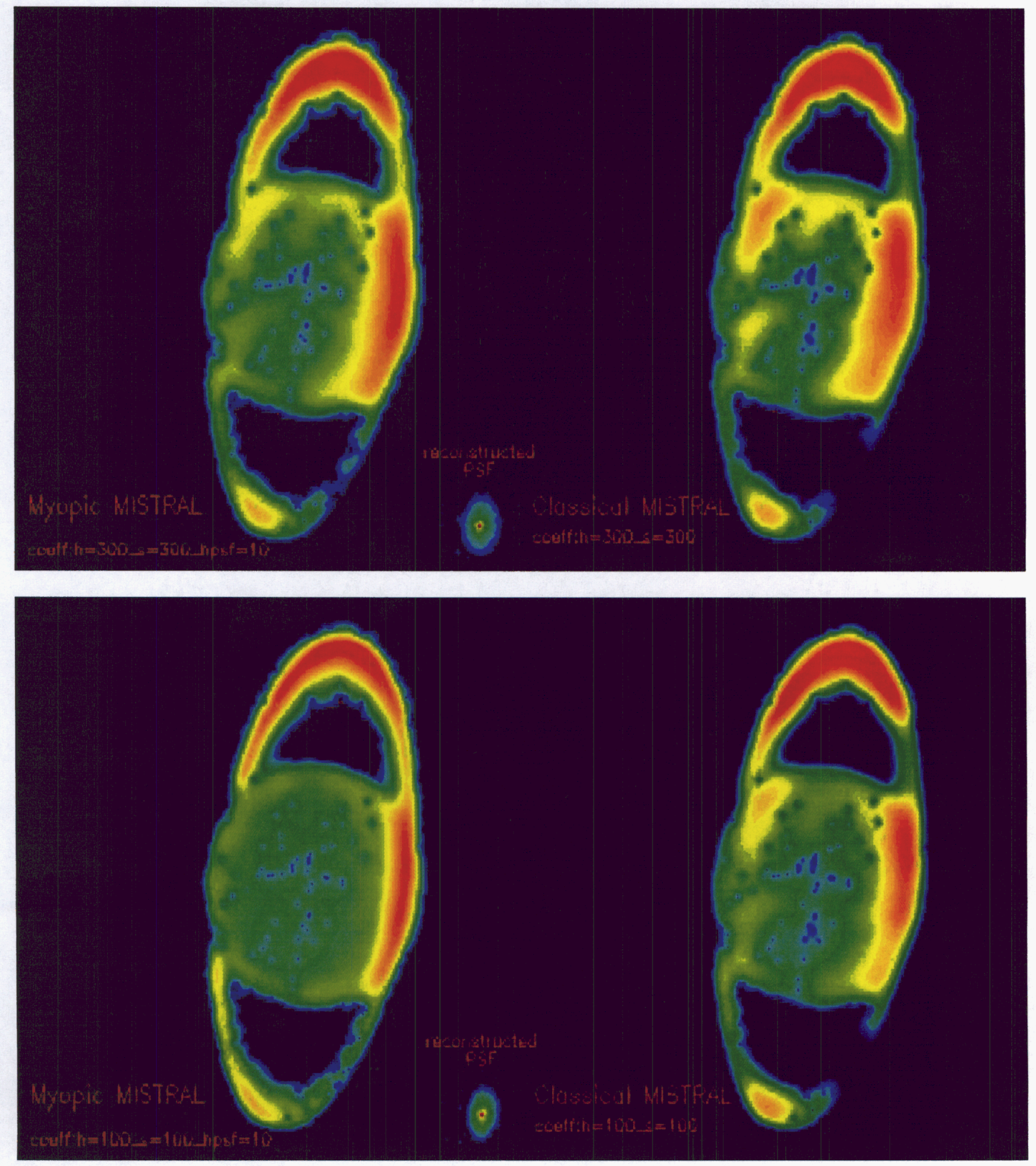


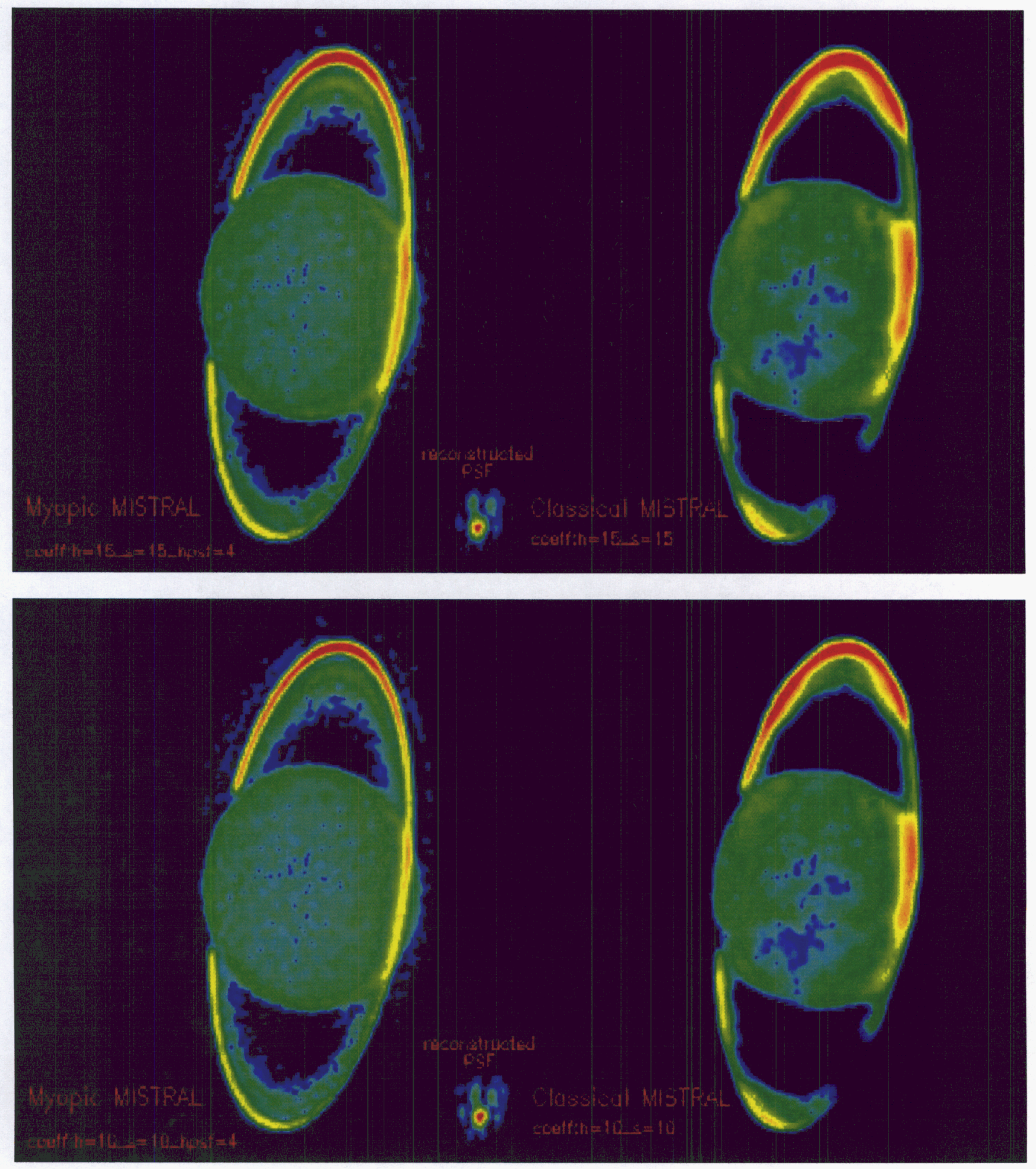

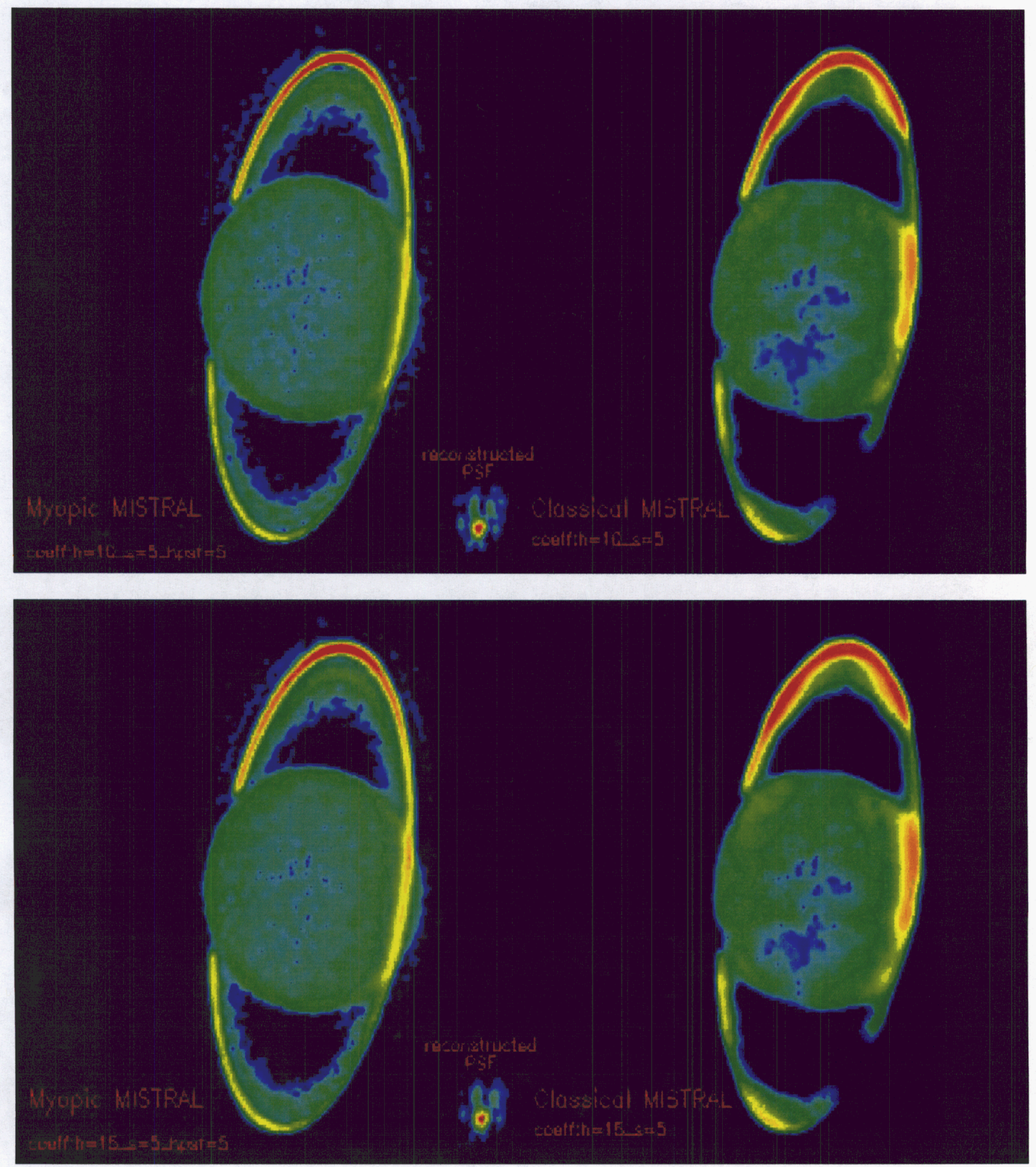

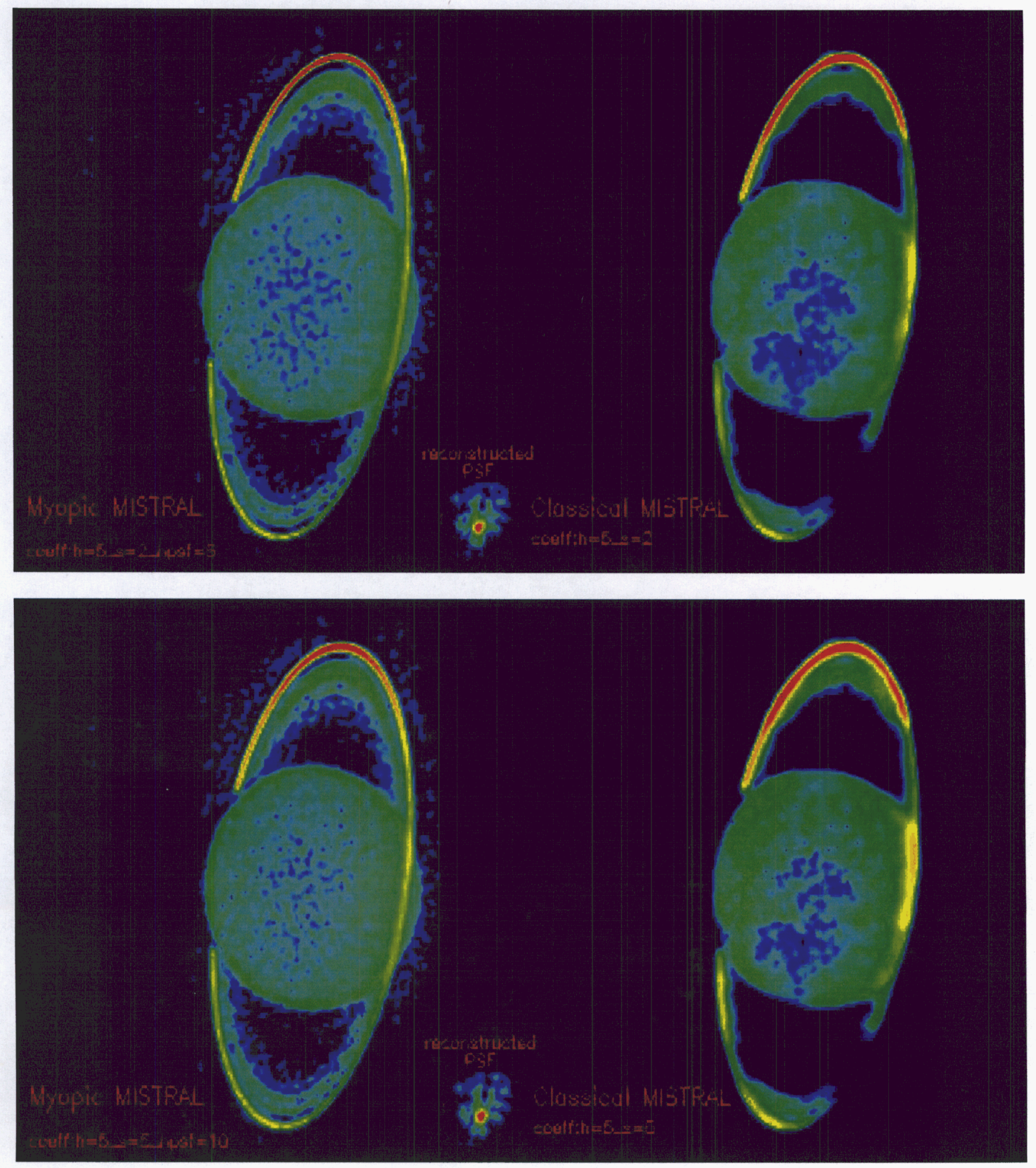

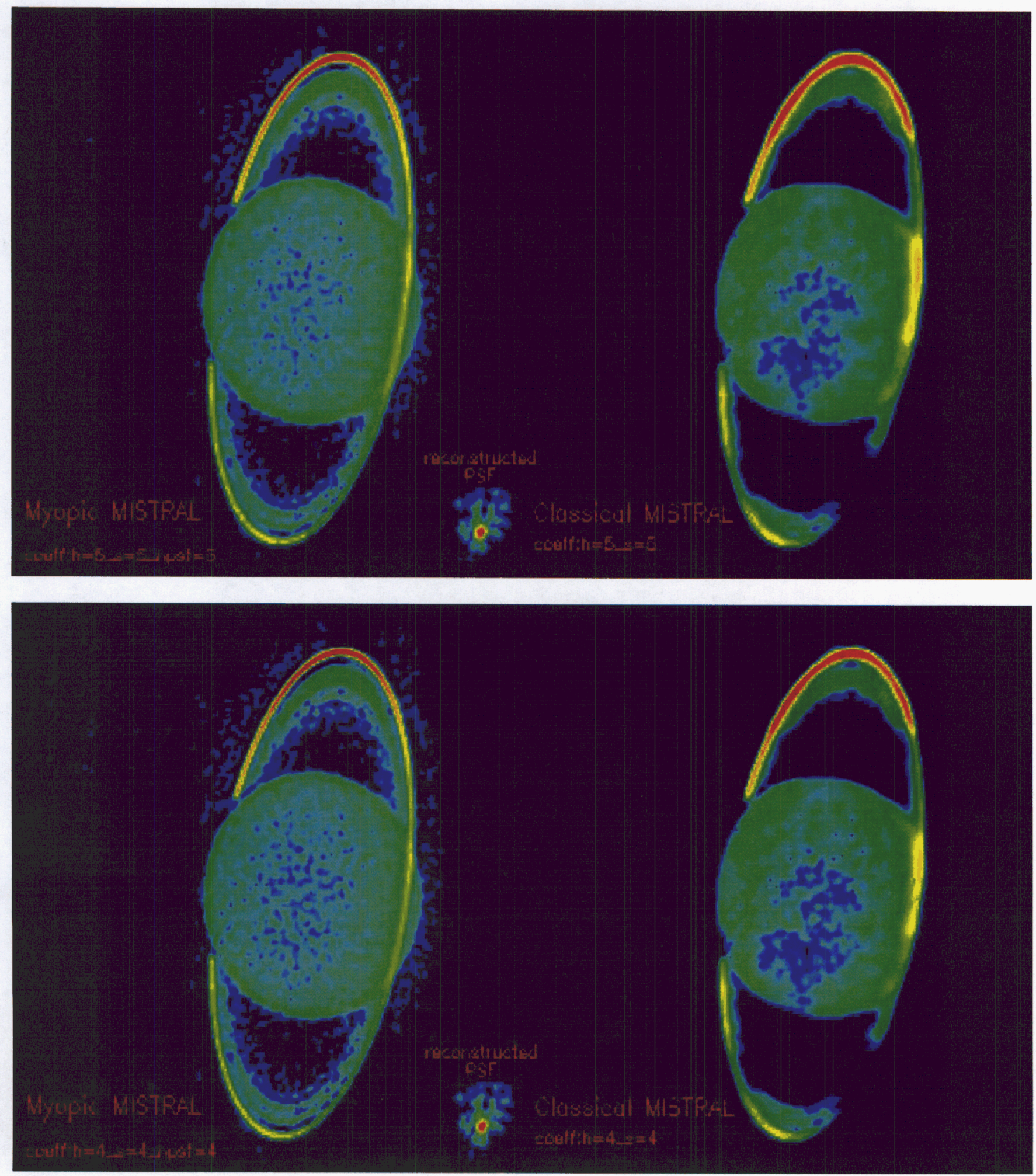

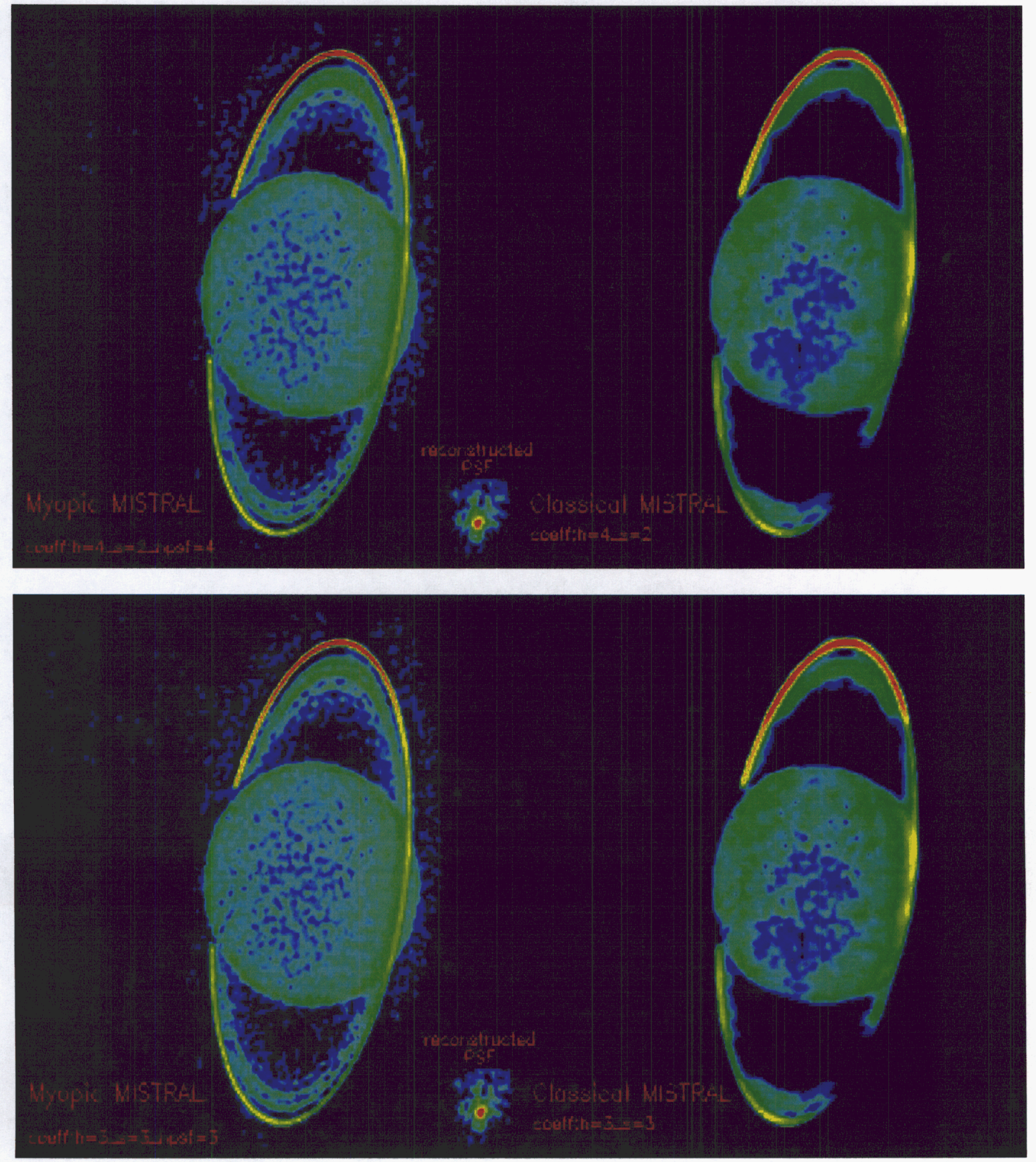

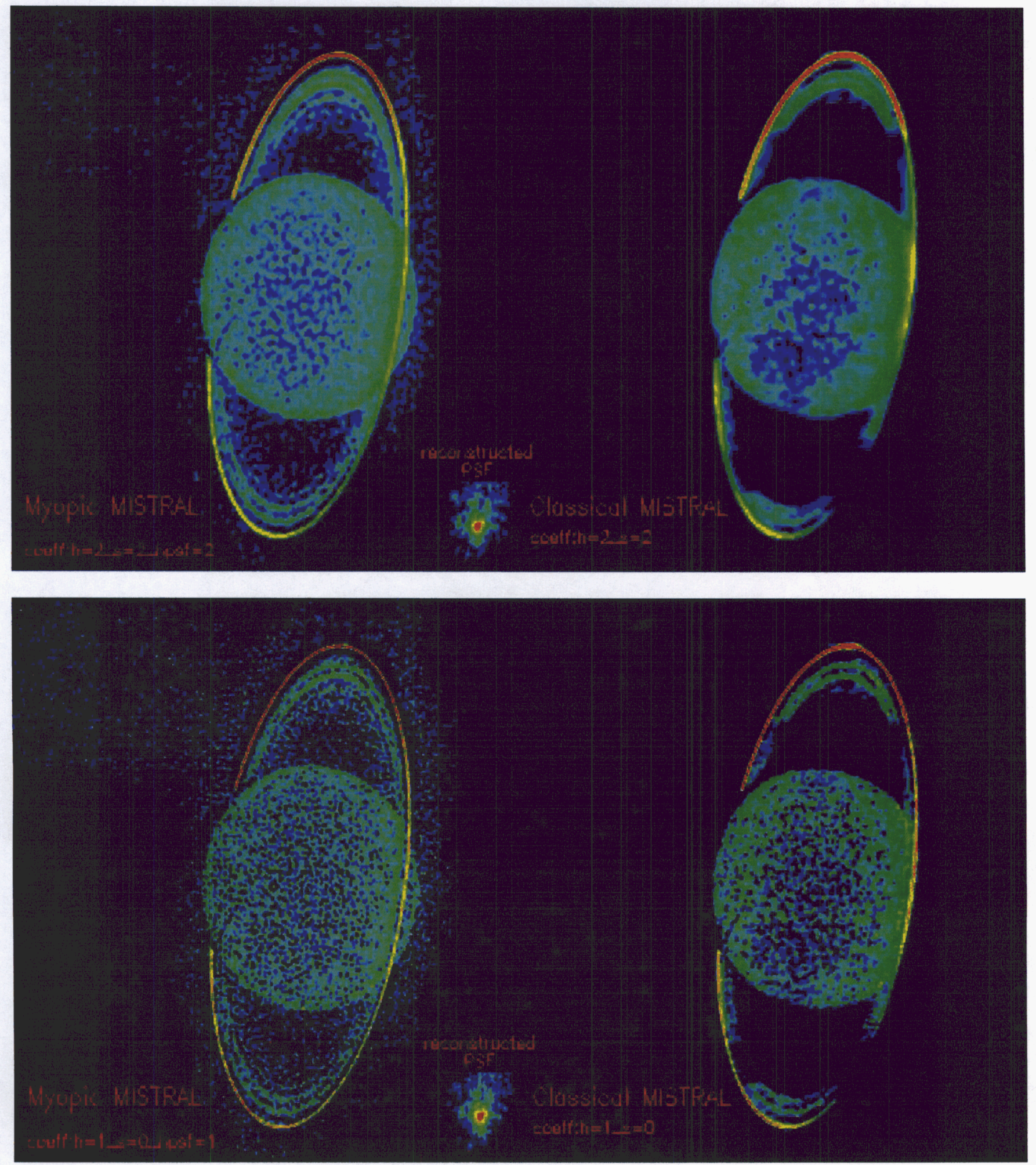

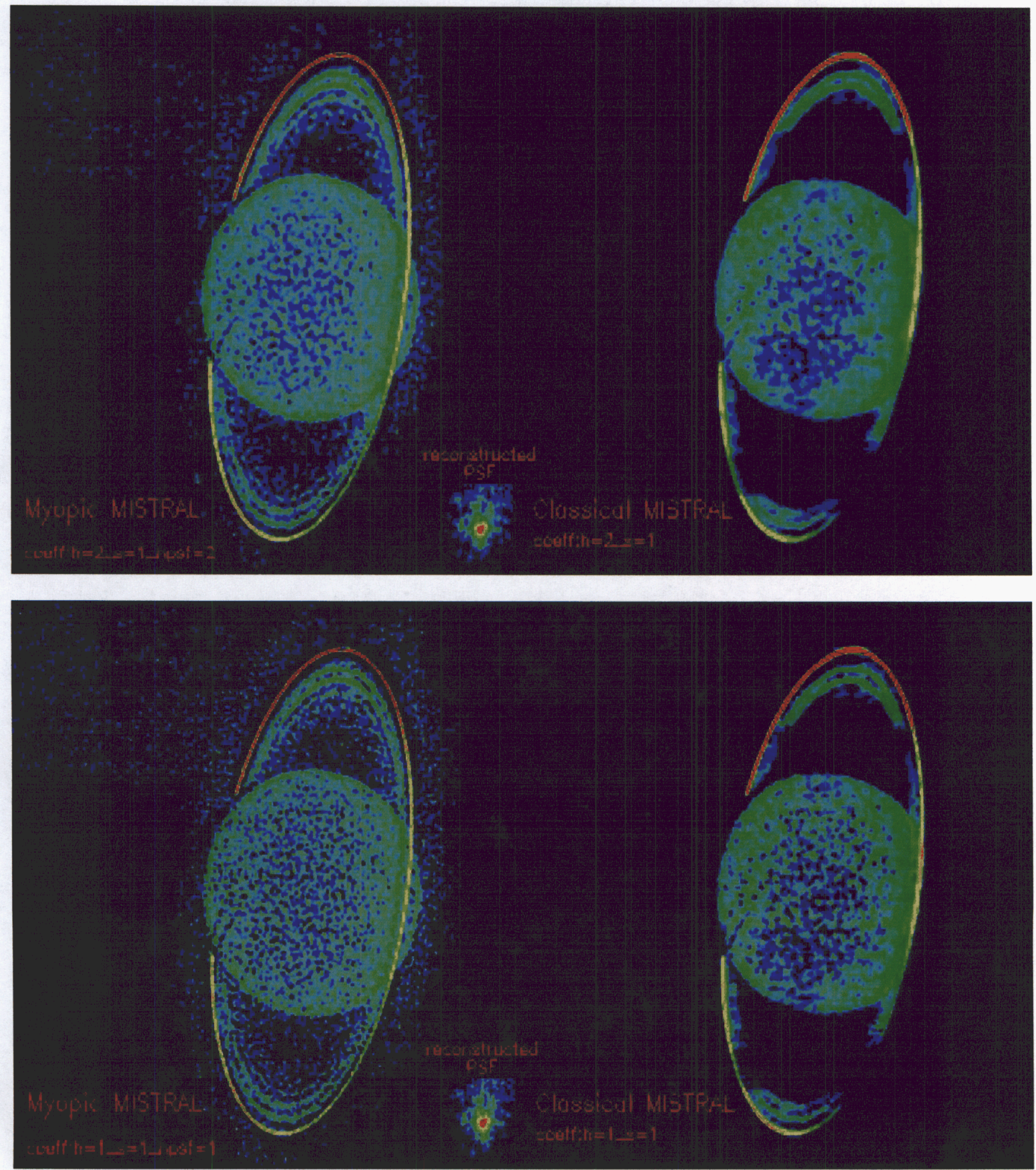

\section{Results and future work}

It is clear that MISTRAL clearly enhances the sharpness of these data. There is still a residual noise on the north part of the image with a brighness similar to the fainter satellite detected (Desdemona) impeding the unambiguous detection of satellites. I do not think this problem can be solved on this data 
since it may come from the non uniform background introduce by the basic data processed.

I am now running the deconvolution of a $1000 * 1000$ pixel frame containing all the internal satellites using the optimal parameter estimated on this smaller image. It will take more than 2 days on Floris to get one deconvolved image.

Deconvolution of $\mathrm{J}$ and $\mathrm{H}$ images will be interesting to get an estimate the color of rings and satellites (see Trilling et al., 2000).

We can perform an astrometrical study of position of the satellites, but we do not have bright references in the field of view (star, bright satellites). Study of Puck position should be valuable (as done in Descamps et al. 2002).

A complete analysis on the ring structure and Epsilon variation of intensity seen on ADONIS and this data should be interesting if real...

Finally, deconvolution of new NIRC2 data (Uranus, Neptune) taken in July 2002 must be done...(when, who?)

Simulation (work of Amanda...)

\section{Franck Marchis}

Last modified: Tue Aug 20 12:21:29 PDT 2002 